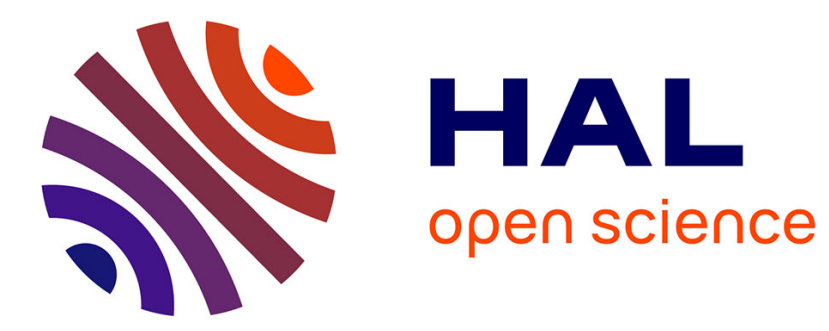

\title{
Automatic guidance of a farm tractor along curved paths, using a unique CP-DGPS
}

Benoît Thuilot, Christophe Cariou, Lionel Cordesses, Philippe Martinet

\section{To cite this version:}

Benoît Thuilot, Christophe Cariou, Lionel Cordesses, Philippe Martinet. Automatic guidance of a farm tractor along curved paths, using a unique CP-DGPS. RSJ/IEEE International Conference on Intelligent Robots and Systems, Nov 2001, Maui, France. pp.674-679, 10.1109/IROS.2001.976247 . hal-02466214

\section{HAL Id: hal-02466214 https://hal.inria.fr/hal-02466214}

Submitted on 4 Feb 2020

HAL is a multi-disciplinary open access archive for the deposit and dissemination of scientific research documents, whether they are published or not. The documents may come from teaching and research institutions in France or abroad, or from public or private research centers.
L'archive ouverte pluridisciplinaire HAL, est destinée au dépôt et à la diffusion de documents scientifiques de niveau recherche, publiés ou non, émanant des établissements d'enseignement et de recherche français ou étrangers, des laboratoires publics ou privés. 


\title{
Automatic guidance of a farm tractor along curved paths, using a unique CP-DGPS
}

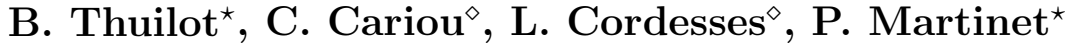 \\ * LASMEA \\ $\checkmark$ Cemagref \\ 24, av. des Landais \\ 63177 Aubière Cedex France \\ Benoit.Thuilot@lasmea.univ-bpclermont.fr \\ BP 50085 - 24, av. des Landais \\ 63172 Aubière Cedex France \\ christophe.cariou@CLERMONT.cemagref.fr
}

\begin{abstract}
Precision agriculture involves very accurate farm vehicle control along recorded paths, which are not necessarily straight lines. In this paper, we investigate the possibility of achieving this task with a CP-DGPS as the unique sensor. The vehicle heading is derived according to a Kalman state reconstructor, and a nonlinear velocity independent control law is designed, relying on chained systems properties. Field experiments, demonstrating the capabilities of our guidance system, are reported and discussed.

Keywords: mobile robots, nonlinear control systems, Kalman state reconstructor, GPS, agriculture.
\end{abstract}

\section{Introduction}

The development of guidance systems for agricultural vehicles receives more and more attention from researchers and manufacturers. The motivations are numerous, since automatic guidance:

- reduces the work arduousness.

- insures an optimal work precision throughout the day and on the whole field. For instance, it enables the exact placement of field inputs (seeds, fertilizers, pesticides,...).

- allows to operate wider tools at higher speeds, and therefore it can increase productivity.

Many experiments have been conducted and reported in the literature. They can be classified into 2 categories, according to the kind of sensor to be used.

The first category uses relative information. Researchers have focused for instance on the video camera sensor [4], [6] and [9]. Two kinds of difficulties arise: the first one is the reference extraction. Extreme conditions of dust, for example, reveal the weakness of the detection side. The other problem arises when the new reference is computed in an iterative way from the previous one. The automatic guidance system can lead to oscillations.

The second category uses absolute information. State of the art GPS (Carrier Phase Differential GPS or CP-DGPS) reach today the realtime centimeter accuracy. This allows the design and implementation of an absolute vehicle guidance system. Promising results have been reported: a GPS and a fiber optic gyroscope (FOG) have been used in [7]. [8] has described a solution with multiple GPS antennas. These multisensors equipments have the advantage to provide control designers with the whole vehicle attitude. However, they are quite expensive. Therefore, we are currently investigating the possibility to control vehicles with a unique GPS. Straight line following, applied to a combine harvester, has been demonstrated in [3]. The aim of this paper is to extend this work to curved path following applications. Our experimental vehicle, depicted on Figure 1, is now a farm tractor.

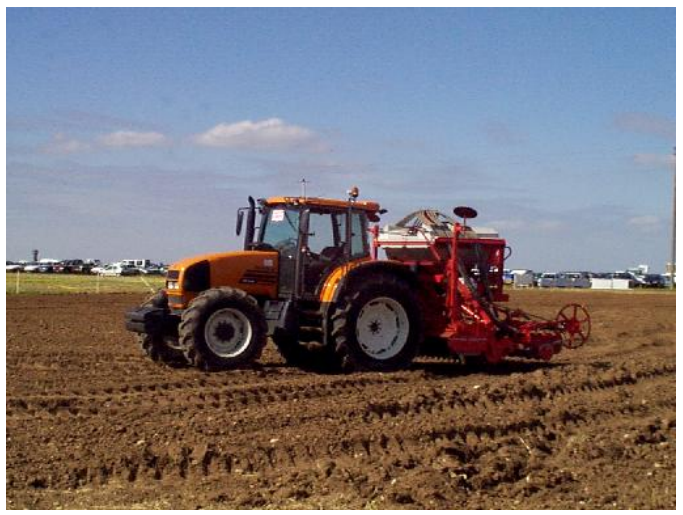

Figure 1: Experimental platform 
We first derive the kinematic model of a farm tractor. Then, the design of a nonlinear, velocity independent, curved path following control law is detailed. Next, the estimation of the tractor heading from GPS information is discussed. Finally experimental results are displayed.

\section{Modelisation}

From a practical point of view, the tractor and the tool can be considered as a tricycle model of length $l$ with a controlled front wheel, see Figure 2.

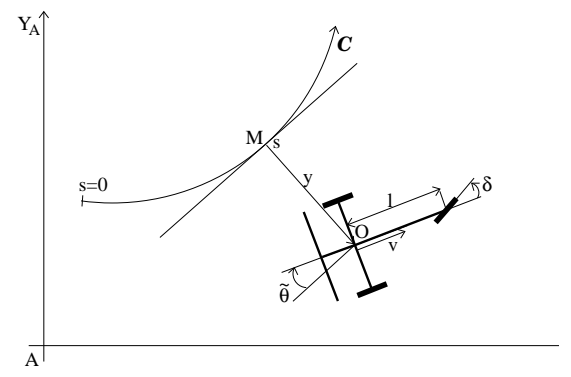

Figure 2: Kinematic model of the farm tractor

The tractor is assumed to move on a horizontal ground. We propose here to describe its configuration with respect to the path $\mathcal{C}$ to be followed, rather than with respect to an absolute frame $\left[A, X_{A}, Y_{A}\right)$. For this purpose, let us denote by $O$ the center of the tractor rear axle, and by $M$, the point on $\mathcal{C}$ which is the closest to $O$ (see Figure 2). The vehicle configuration is then described without ambiguity by the state vector $X=(s, y, \tilde{\theta})^{T}$ with:

- $s$ : the curvilinear abscissa of point $M$ along $\mathcal{C}$,

- $y$ : the lateral deviation of the tractor with respect to $\mathcal{C}$,

- $\tilde{\theta}$ : the angular deviation of the tractor with respect to the tangent to $\mathcal{C}$ at point $M$.

Two control variables are available, namely $U=(v, \delta)^{T}$ :

- $v$ : linear velocity at point $O$ (the control variable is actually the rear wheels rotation velocity, from which the value of $v$ can be inferred).

- $\delta$ : orientation of the front wheel.

We have derived the vehicle kinematic model according to the following assumptions:

- the tractor and the tool are a unique rigid body,

- the tractor moves according to pure rolling and non slipping conditions.
They are quite common among mobile robots community: the former implies that an instantaneous rotation center exists. The latter imposes that the linear velocity vector at point $O$ is directed along the vehicle axle. Under such assumptions, we obtain, see [2]:

$$
\left\{\begin{aligned}
\dot{s} & =v \frac{\cos \tilde{\theta}}{1-c(s) y} \\
\dot{y} & =v \sin \tilde{\theta} \\
\dot{\tilde{\theta}} & =v\left(\frac{\tan \delta}{l}-\frac{c(s) \cos \tilde{\theta}}{1-c(s) y}\right)
\end{aligned}\right.
$$

where $c(s)$ denotes the curvature of path $\mathcal{C}$ at the curvilinear abscissa $s$ (i.e. at point $M$ ).

It can be noticed, that the model (1) becomes singular when $y=\frac{1}{c(s)}$, i.e. when the point $O$ is located at the center of curvature of the reference path. This problem is not encountered in practical situations: on one hand, the path curvature is small, and on the other hand, the farm tractor remains close to $\mathcal{C}$.

\section{Control law design}

The control objective is to ensure the convergence of the tractor to the reference path $\mathcal{C}$. In view of section 2 , we want state variables $y$ and $\tilde{\theta}$ to be brought and kept equal to 0 . Moreover, control law performances are expected to be independent from tractor velocity $v$. This control variable is here considered as a parameter whose value will be managed according to the application. $v$ may be constant or time-varying.

The tractor model (1) is clearly nonlinear. In [1], the authors propose to linearize it around the equilibrium $y=\tilde{\theta}=0$, in order that celebrated linear systems theory could be used. In this case, control design does not rely on the actual tractor model, but on an approximated one. Recent advances in Control theory have established that mobile robots models can be converted into almost linear models, namely chained forms, in an exact way, see [10] for instance. Such an approach is attracting since it allows us to use, for a large part, linear systems theory, while still relying upon the actual nonlinear tractor model. It is detailed hereafter.

The general chained form dedicated to systems with three states and two inputs is written as (see [10]):

$$
\left\{\begin{array}{l}
\dot{a}_{1}=m_{1} \\
\dot{a}_{2}=a_{3} m_{1} \\
\dot{a}_{3}=m_{2}
\end{array}\right.
$$

with $A=\left(a_{1}, a_{2}, a_{3}\right)^{T}$ and $M=\left(m_{1}, m_{2}\right)^{T}$ respectively the state and control vectors. In order to point out that a chained system is almost linear, just replace 
the time derivative by a derivation with respect to the state variable $a_{1}$. Using the notations:

$$
\frac{d}{d a_{1}} a_{i}=a_{i}^{\prime} \quad \text { and } \quad m_{3}=\frac{m_{2}}{m_{1}}
$$

the chained form (2) can be rewritten:

$$
\left\{\begin{array}{l}
a_{1}^{\prime}=1 \\
a_{2}^{\prime}=a_{3} \\
a_{3}^{\prime}=m_{3}
\end{array}\right.
$$

The last 2 equations of system (4) constitute clearly a linear system. More generally, a $n$-dimensional chained system exhibits a $n$-1-dimensional linear subsystem.

Let us now convert tractor model (1) into chained form. Since control law performances are expected to be independent from the tractor velocity, the variable $a_{1}$, which drives the evolution of the linear system (4), should be homogeneous at the distance covered by the tractor. A natural choice is then:

$$
a_{1}=s
$$

Consequently, variables $a_{2}$ and $a_{3}$ have to be related to $y$ and $\tilde{\theta}$ in an invertible way. For the sake of simplicity, let us choose :

$$
a_{2}=y
$$

Straightforward computations show then that the nonlinear tractor model (1) can actually be converted into chained forms (2) or (4) from the starting choices (5)(6). More precisely, we can show successively that:

$$
\begin{aligned}
m_{1} \triangleq \dot{a}_{1}= & v \frac{\cos \tilde{\theta}}{1-c(s) y} \\
a_{3} \triangleq \frac{\dot{a}_{2}}{m_{1}}= & (1-c(s) y) \tan \tilde{\theta} \\
m_{2} \triangleq \dot{a}_{3}= & \frac{d}{d t}((1-c(s) y) \tan \tilde{\theta}) \\
= & -c(s) v \sin \tilde{\theta} \tan \tilde{\theta}-\frac{d c(s)}{d s} \frac{v \cos \tilde{\theta}}{1-c(s) y} \tan \tilde{\theta} y \\
& +v \frac{(1-c(s) y)}{\cos ^{2} \tilde{\theta}}\left(\frac{\tan \delta}{l}-c(s) \frac{\cos \tilde{\theta}}{1-c(s) y}\right)
\end{aligned}
$$

In the sequel, state and control transformations are denoted respectively $\Theta(X)$ and $\Upsilon(U, X)$ :

$$
\begin{gathered}
A=\Theta(X) \text { defined by (5), (6) and (8) } \\
M=\Upsilon(U, X) \text { defined by (7) and (9) }
\end{gathered}
$$

These transformations are invertible as long as $y \neq$ $\frac{1}{c(s)}$ (model singularity), $v \neq 0$, and $\tilde{\theta} \neq \frac{\pi}{2}[\pi]$. From a practical point of view, once properly initialized, the guided tractor respects these conditions.
Control design can now be completed in a very simple way: since chained form (4) is linear, we are led to choose the following virtual control law:

$$
m_{3}=-K_{d} a_{3}-K_{p} a_{2} \quad\left(K_{p}, K_{d}\right) \in \mathcal{R}^{+2}
$$

As a matter of fact, reporting (12) in (4), leads to:

$$
a_{2}^{\prime \prime}+K_{d} a_{2}^{\prime}+K_{p} a_{2}=0
$$

which implies that both $a_{2}$ and $a_{3}$ converge to zero. The same conclusion holds for $y$ and $\tilde{\theta}$ in view of (10). Reference path following is therefore achieved. Moreover, since the evolution of the error dynamics (13) is driven by $a_{1}=s$, the gains $\left(K_{d}, K_{p}\right)$ impose a settling distance instead of a settling time. Consequently, for a given initial error, the tractor trajectory will be identical, whatever the value of $v$ is, and even if $v$ is time-varying. Control law performances are therefore velocity independent, as desired.

Ultimately, reporting (12) into (11) provides us with the actual control law expression:

$$
\begin{aligned}
\delta(y, \tilde{\theta})= & \arctan \left(l \left[\frac { \operatorname { c o s } ^ { 3 } \tilde { \theta } } { ( 1 - c ( s ) y ) ^ { 2 } } \left(\frac{d c(s)}{d s} y \tan \tilde{\theta}\right.\right.\right. \\
& -K_{d}(1-c(s) y) \tan \tilde{\theta}-K_{p} y \\
& \left.\left.\left.+c(s)(1-c(s) y) \tan ^{2} \tilde{\theta}\right)+\frac{c(s) \cos \tilde{\theta}}{1-c(s) y}\right]\right)
\end{aligned}
$$

In many applications, the reference path $\mathcal{C}$ is a straight line, i.e. $c(s)=0$. The expression of the control law (14) turns then simpler:

$$
\delta(y, \tilde{\theta})=\arctan \left(l \cos ^{3} \tilde{\theta}\left(-K_{d} \tan \tilde{\theta}-K_{p} y\right)\right)
$$

Since control law (14) is not bounded, actuator saturation could occur. We are allowed to bound the virtual control law (12) to any arbitrary value: since chained form (4) consists in a double integrator, its asymptotic stability is still insured, see [11]. Nevertheless, in view of (14), the boundedness of $m_{3}$ leads to those of $\delta$ only if the reference path curvature $c(s)$ exhibits some good properties. They are of course satisfied in the straight line case, i.e. when $c(s)=0$. In the forthcoming experimentations, control law (12) has been saturated by means of a sigmoid function:

$$
m_{3, s a t}\left(a_{2}, a_{3}\right)=K \frac{1-e^{-k m_{3}\left(a_{2}, a_{3}\right)}}{1+e^{-k m_{3}\left(a_{2}, a_{3}\right)}}
$$

Once saturated virtual control law (16) has been reported into (11), parameter $K$ can be tuned so as to ensure that $\max _{\forall y, \forall \tilde{\theta}}|\delta(y, \tilde{\theta})|$ remains inferior to actuator limitation $\delta_{\max }$. Finally, we must choose $k=\frac{2}{K}$ in order that $m_{3, \text { sat }}\left(a_{2}, a_{3}\right) \approx m_{3}\left(a_{2}, a_{3}\right)$ when $a_{2}$ and $a_{3}$ are small. 


\section{State reconstruction}

The only exteroceptive sensor embarked on the tractor is a CP-DGPS. It has been located on the top of the cabin, straight up the point $O$. Absolute position and velocity of that point, which is the tractor control point (see Figure 2), are then available in a direct way. In addition, this location is on the highest part of the tractor, and therefore the suitest place to see as many satellites as possible.

Control law (14) requires realtime measurement of $y$ and $\tilde{\theta}$. The value of the former variable can be obtained straightforwardly: from the absolute position of point $O$, provided by the GPS, and the knowledge of the reference path $\mathcal{C}$, we can infer the location of point $M$, and therefore the value of $y$.

The value of $\tilde{\theta}$ is more complex to derive. Under the pure rolling and non-slipping assumptions, the tractor heading with respect to the absolute frame $\left[A, X_{A}, Y_{A}\right)$, denoted $\theta$, can be derived from the absolute velocity of point $O$, denoted $\left(V_{0}, X_{A}, V_{0, Y_{A}}\right)$, also provided by the GPS:

$$
\theta= \begin{cases}\arctan \frac{V_{0, Y_{A}}}{V_{0}, X_{A}} & \text { if } V_{0, X_{A}} \neq 0, \\ \operatorname{sign}\left(V_{0}, Y_{A}\right) \frac{\pi}{2} & \text { if } V_{0, X_{A}}=0\end{cases}
$$

Therefore, the value of $\tilde{\theta}$ can also be obtained by a direct measurement:

$$
\tilde{\theta}=\theta-\theta_{d}(s)
$$

where $\theta_{d}(s)$ denotes the heading of the reference path $\mathcal{C}$ at the curvilinear abscissa $s$ (i.e. at point $M$ ).

Unfortunately, experiments have shown that a direct measurement (18) provides us with prohibitive noisy values of $\tilde{\theta}$. A low pass filter could be considered. However, since the tractor model is available, a suitable alternative consists in using it through a Kalman state reconstructor. Discretizing the third equation of tractor model (1) leads to:

$$
\tilde{\theta}_{k+1}=\tilde{\theta}_{k}+\frac{v T}{l} \tan \delta_{k}
$$

where $T$ is the sampling period. Celebrated Kalman state reconstructor equations, when applied to system (19), are then written as (see for instance [5]):

$$
\left\{\begin{array}{l}
\overline{\tilde{\theta}}_{k}=\hat{\tilde{\theta}}_{k-1}+\frac{v T}{l} \tan \delta\left(y_{k-1}, \hat{\tilde{\theta}}_{k-1}\right) \\
\tilde{\tilde{\theta}}_{k}=\tilde{\tilde{\theta}}_{k}+L\left(\tilde{\theta}_{k}^{m}-\tilde{\tilde{\theta}}_{k}\right)
\end{array}\right.
$$

$L$ is the scalar Kalman gain, to be chosen with respect to the sensor noise features. $\tilde{\theta}_{k}^{m}$ denotes the direct measurement of $\tilde{\theta}$, according to (18), at time $t_{k}=k T$. $\hat{\tilde{\theta}}_{k}$ is its filtered value at the same time, to be used in control law (14).

\section{$5 \quad$ Experimentations}

The farm tractor and its tool are depicted on Figure 1. The CP-DGPS receiver is a Dassault-Sercel dual frequency "Aquarius 5002" unit. Its claimed accuracy is about 1 centimeter, and its upper sampling frequency is 10 Hertz. The control law (14) has been implemented in high level language $(\mathrm{C}++)$ on a Pentium based computer.

\section{$5.1 \quad$ Step responses}

Although a step path is not common in agriculture, we have used the step response to check the behaviour of our closed loop system.

The reference path consists in a 2 meters step. Control law parameters $\left(K_{d}, K_{p}\right)$ have been tuned so as to impose that the error dynamics (13) presents a 15 meters settling distance ${ }^{1}$, while Kalman parameter $L$ has been adjusted with the value 0.08 , in view of the sensor noise features. Three experiments have been carried out: in the two first, the tractor velocity was constant, $v=4 \mathrm{~km} \cdot \mathrm{h}^{-1}$, and then $v=8 \mathrm{~km} \cdot \mathrm{h}^{-1}$, while in the last one, the velocity has been increased linearly from $v=4 \mathrm{~km} \cdot \mathrm{h}^{-1}$ to $v=8 \mathrm{~km} \cdot \mathrm{h}^{-1}$. The experimental results are depicted on Figure 3 and 4 .
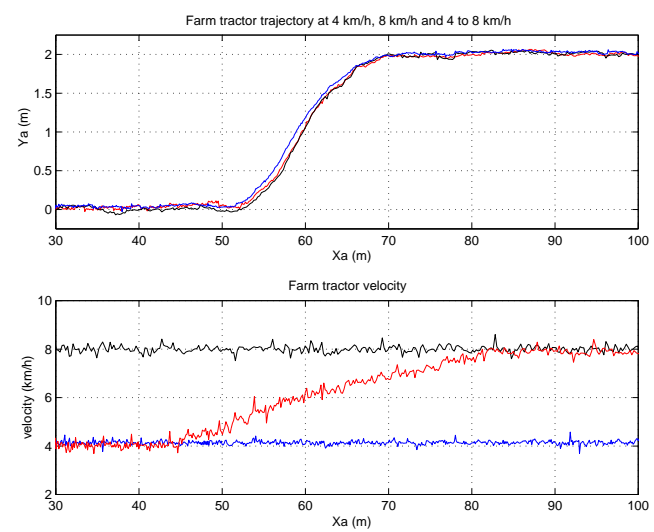

Figure 3: Step responses at different velocities

On Figure 3, the upper plot shows the three step responses, while the lower plot depicts the tractor raw velocity, as computed by the GPS receiver. One can check that the three step responses almost perfectly overlap: as expected, the trajectory is independent from tractor velocity, even when $v$ is varying during the step. Moreover, the 15 meter settling distance, specified when tuning the control parameters,

\footnotetext{
${ }^{1}$ We have chosen $\left(K_{d}, K_{p}\right)=(0.6,0.09)$ in order that the error dynamics (13) presents a double pole located at the value 0.3 . Linear control tools ensure then that the settling distance is 15 meters, as it was specified.
} 
is actually achieved. If we investigate path following accuracy, the results appear also quite satisfactory: once the tractor is following a straight line (i.e. when $x_{a}>70$ meters), the bias $\mu_{y}$ between the tractor trajectory and the reference path, as well as the tractor standard deviation from the mean trajectory $\sigma_{y}$, are both very small: $\mu_{y}<2.7 \mathrm{~cm}$ and $\sigma_{y}<3.1 \mathrm{~cm}$.

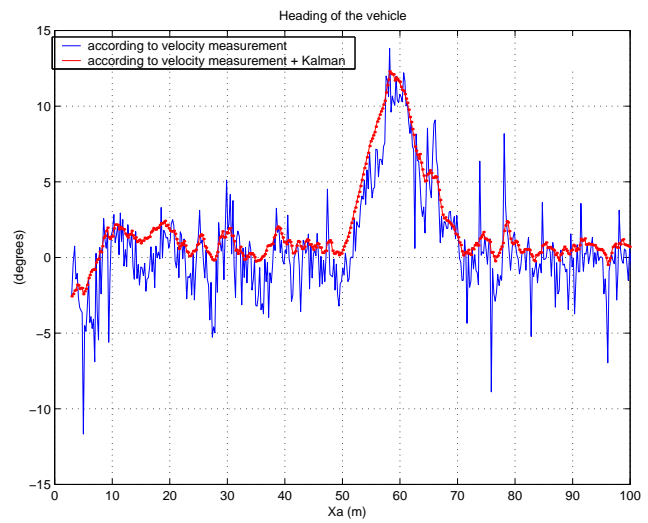

Figure 4: Estimation of heading error $\tilde{\theta}$

The values of state variable $\tilde{\theta}$, required in control laws (14) or (15), can be obtained either by a direct measurement of tractor velocity, according to relations (17) and (18), or by filtering this direct measurement according to the Kalman state reconstructor (20). The values of $\tilde{\theta}$ returned by these two approaches, have been recorded during a step response with a constant tractor velocity $v=8 \mathrm{~km} \cdot \mathrm{h}^{-1}$, and are displayed on Figure 4. It clearly highlights the need for filtering the values of $\tilde{\theta}$ returned by a direct measurement, and establishes the relevancy of the Kalman state reconstructor (20): the amplitude of $\tilde{\theta}$ oscillations, as well as theirs frequencies, are significantly reduced. For instance, once the tractor is following a straight line (i.e. when $x_{a}>70$ meters), the standard deviation from the mean decreases from 1.71 to 0.48 when the Kalman state reconstructor is used.

\subsection{Curved path following}

The first experiments reported below consist in a sinusoidal path following. Sine curves are definitely not common trajectories in agricultural tasks. Nevertheless, they are significantly different from straight lines, and therefore can be seen as a convincing test when investigating the performances of curved path following control law (14).

Period and peak to peak amplitude of the sinusoidal reference path are respectively 20 meters and 60 centimeters. The values of the control and Kalman pa- rameters $K_{d}, K_{p}$ and $L$ are identical to those used in step response experiments. At initial time, the tractor is 60 centimeters far from the reference path. Its velocity is constant: $v=6 \mathrm{~km} \cdot \mathrm{h}^{-1}$.

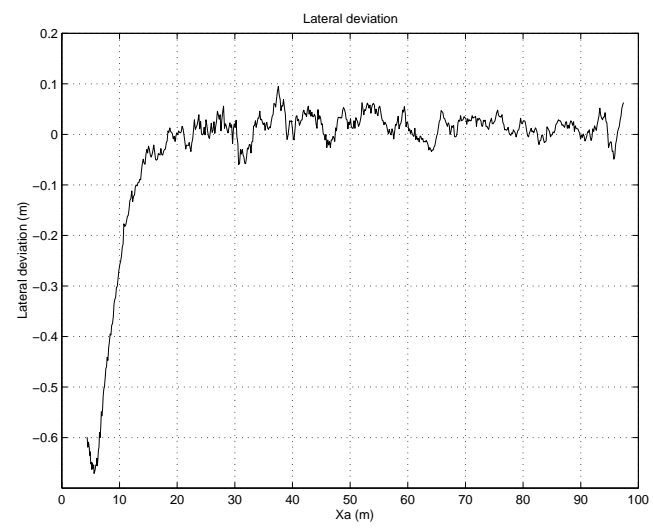

Figure 5: Lateral deviation with respect to a sinusoidal reference path

The evolution of the tractor lateral deviation with respect to the sinusoidal reference path is depicted on Figure 5. One can check that control law (14) leads to the same performances, irrespectively of the reference path features: the settling distance and the statistical variables $\mu_{y}$ and $\sigma_{y}$ recorded during this curved path following experiment are of the same order of magnitude as those previously recorded during step response experiments.

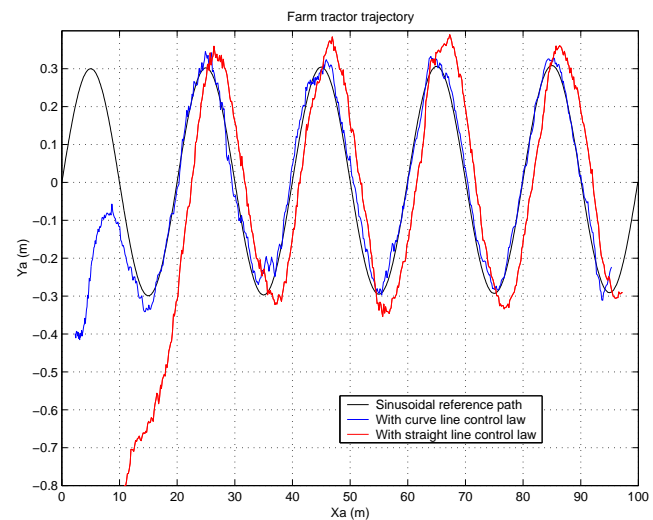

Figure 6: Tractor trajectories during a sine curve following: capabilities of control laws (14) and (15)

Curved path control law (14) can be gracefully degraded to the case of straight lines following by imposing $c(s)=0$ : we then obtain the much simpler expression (15). In order to investigate the actual contribution of the reference path curvature $c(s)$, we have also experimented sine curve following with the simplified 
control law (15). Reference path, tractor trajectory obtained with that law, and those obtained with the entire control law (14) are displayed on Figure 6. We observe that, without the reference path curvature information, the tractor follows the reference path with an 1.5 meter offset, and moreover presents large excursions when the values of $c(s)$ are the highest. This clearly establishes the imperative need to take into account the curvature of the reference path when designing the control law.

Of course, we have also investigated control law (14) capabilities when the path to be followed is consistent with common agricultural tasks. The tractor has first been driven on a field by a human operator. The path was roughly made of straight lines and semicircles. This path has then been replayed several times in an automatic way. Examination of the results shows that the tractor trajectories almost overlap the reference path. A statistical analysis still provides us with quite satisfactory results: $\mu_{y}=3 \mathrm{~cm}$ and $\sigma_{y}=5 \mathrm{~cm}$. Nevertheless, it can be noticed that, on few parts of the reference path, the lateral deviation climbs up to large values $(18.4 \mathrm{~cm}$ in the worst case). The explanation that can be advanced is that the reference path curvature, in these locations, is very high, thus leading to a control $\delta$ varying very quickly. This has led us to incriminate the low level servoing system, since it has not been optimized for fast tracking: neither the hydraulic components nor the controller - off the shelves devices - can achieve very high performances.

Finally, during Innov'Agri 2000 demonstrations (a French Show where new agricultural systems are presented), the tractor has replayed a complex curved path more than a hundred times without any driver abroad, establishing the system reliability.

\section{Conclusion}

The purpose of this work was to achieve curved path following from a unique CP-DGPS sensor, within the framework of agricultural applications. A Kalman state reconstructor has been derived in order to estimate the tractor heading, and a nonlinear velocity independent control law has been designed, relying upon recent developments in Control theory, precisely "chained systems" properties. Several experiments are reported, and display the control law capabilites.

Although results are quite satisfactory on an almost flat ground, further research has to be done to take into account for slope. Another important issue is to build a better hydraulic servoing system, in order to decrease both latency and settling time.

\section{References}

[1] T. Bell, M. O'Connor, V. K. Jones, A. Rekow, G. Elkaim, and B. Parkinson. Realistic autofarming closed-loop tractor control over irregular paths using kinematic GPS. In European Conf. on Precision Agriculture, 1997.

[2] C. Canudas de Wit, B. Siciliano, and G. Bastin eds. Theory of robot control. Springer-Verlag, 1996.

[3] L. Cordesses, P. Martinet, B. Thuilot, and M. Berducat. GPS-based control of a land vehicle. In IEEE Intern. Symp. on Automation and Robotics in Construction, pages 41-46, 1999.

[4] C. Debain, T. Chateau, M. Berducat, P. Martinet, and P. Bonton. An help guidance system for agricultural vehicles. Computers and Electronics in Agriculture, 25(1-2):29-51, January 2000.

[5] A. Gelb. Applied optimal estimation. MIT Press, 1974.

[6] D. Khadraoui, C. Debain, R. Rouveure, P. Martinet, P. Bonton, and J. Gallice. Vision based control in driving assistance of agricultural vehicles. International Journal of Robotics Research, 17(10):1040-1054, October 1998.

[7] Y. Nagasaka, R. Otani, K. Shigeta, and K. Taniwaki. Automated operation in paddy fields with a fiber optic gyro sensor and GPS. In Intern. Workshop on Robotics and Automated Machinery for Bio-Productions, pages 21-26, 1997.

[8] M. O'Connor, G. Elkaim, T. Bell, and B. Parkinson. Automatic steering of a farm vehicle using GPS. In Intern. Conf. on Precision Agriculture 1996, pages 767-777, 1996.

[9] M. Ollis and A. Stentz. Vision based perception for an automated harvester. In Proceedings of the IEEE Intern. Conf. on Intelligent Robots and Systems (IROS'97), volume 2, pages 1838-1844, Grenoble, France, 7-11 September 1997.

[10] C. Samson. Control of chained systems. Application to path following and time-varying point stabilization of mobile robots. IEEE Trans. on Automatic Control, 40(1):64-77, January 1995.

[11] H. Sussmann, E. Sontag, and Y. Yang. A general result on the stabilization of linear systems using bounded controls. IEEE Trans. on Automatic Control, 39(12):2411-2425, January 1994. 